\title{
Research on Energy-saving Control of Three-Phase Asynchronous Motor
}

\author{
Lei Bai \\ BeiHua University, Jilin City, 132001 \\ 82295856@QQ. Com
}

Keywords: Three-phase asynchronous; Optimization; Energy-saving control; Voltage regulator

\begin{abstract}
The energy-saving optimization of three-phase asynchronous motor is studied in this paper through the analyses of its working principle and structure. Via a three-phase AC regulator of neutral line-free star connection, six thyristors VT1 and VT2, VT3 and VT4, and VT5 and VT6 of the regulating model are connected reversely in pair so as to determine the optimum efficiency and power factor, and further produce the technique of realizing the minimum energy consumption and the optimum voltage regulation and control through the automatic minimum energy consumption optimizing control system. The simulation results show that the optimization interval is $[160 \mathrm{~V}$, $220 \mathrm{~V}]$, and the energy saving rate is lifted to $68.5 \%$ after the installation of the optimizing and energy-saving module.
\end{abstract}

\section{Introduction}

AC asynchronous motor finds wide applications in daily life[1] for its simple structure, low price, durability, and operability in harsh working environments. It is of great significance for environmental protection to study structure principle and energy-saving technique of AC asynchronous motor[2]. The paper studies its energy-saving control through the analysis of structure and working principle.

\section{Structure of Three-Phase Asynchronous Motors}

A three-phase asynchronous motor is composed of rotor, stator, rotor coil, stator coil and some accessories such as junction box, bearing, fan blade, and end cap[3]. A stator consists of motor base, stator winding, and stator core. The motor base is mainly to protect and fix stator winding and stator core, and also support end cap[4-6]. The stator core is a part of the motor magnetic circuit, and there is a groove on the inner surface of the core for the placement of three-phase winding. The stator core is placed in the base. The stator winding is made of insulated conductors through three-phase winding, and installed in the core groove of stator. When there is the passage of three-phase alternating current, rotating magnetic field will be produced[7]. The rotor produces induced current, and is driven to rotate and generate mechanical power output. The rotor is composed of fan blade, rotor winding, rotor core, and rotating shaft. The rotor winding is embedded in the groove on the outer circle of the punching plate, and the groove is cast with cage bar. Rotor winding includes cage bar and end rings at its two ends. Cage bar and fan are cast into a whole. The axle is made of carbon steel, supported with bearing at both ends[8].

\section{Working Principle of Three-Phase Asynchronous Motor}

Rotating Magnetic Field. Being special, stator winding of the motor is a kind of three-phase star-like load with symmetrical structure. When its head end is connected with a symmetrical three-phase power supply, three-phase symmetrical current can be generated. The phase difference between two power supplies of each phase is $2 \pi / 3$, and it can be deduced from the magnetic effect of electric current that all windings may produce a magnetic field with sinusoidal variations since they, with sinusoidal current, produce magnetic field with two rotating poles. In other words, when the magnetic field has the rotation of $2 \pi$ angle, sinusoidal current will have the rotation of the 
same angle. Therefore, a rotating magnetic field is produced, and its electrical angle is identical with sinusoidal current in variation. The rotational speed of the rotating magnetic field determines the number of pole pairs. When $P=1$, electrical frequency of sinusoidal alternating current is $50 \mathrm{HZ}$, and rotational speed is 50 circles/second, $n=50 \times 60 \mathrm{r} / \mathrm{min}=3000 \mathrm{r} / \mathrm{min}$; when $P=2$, the variation of the magnetic field is $1 / 2$ circle after alternating current rotates for one circle[9].

Working Principle. The motor is connected with three-phase symmetrical current, and there will be rotating magnetic field produced in stator space. If the magnetic field is rotating clockwise, the rotor will have relative motion in the magnetic field. The corresponding induced electromotive force and current will be produced with the cutting of magnetic line of force, and the acting force of the magnetic field will be exerted on the rotor after winding generation. Under the effect of magnetic torque, the rotor will rotate along the direction of the magnetic field. The rotor must rotate slower than the magnetic field. If their rotational speeds are the same, there will be no relative motion between the magnetic field and rotor. Therefore, the rotor conductor will not have magnetic line cutting, so will rotor current, induced electromotive force, and electromagnetic torque. The rotor will not rotate as well. Hence, the magnetic field and rotor should have the given difference of rotational speeds, which means that their rotational speeds should be different[10].

\section{Energy-saving Control of Three-Phase Asynchronous Motor}

Accurate Online Test of Stator Current. The motor can save more energy if stator current can be tested accurately. For an asynchronous motor, noise will interfere with harmonic wave, thus affecting the accuracy of stator current signal. The output of optimizing and energy-saving module has the direct bearing on the accuracy of stator current signal, and further affects the overall energy-saving effect of the motor. In the research, the accuracy of stator current signal is determined through sliding average value sampling module and low-pass filtering module. Energy-saving control of the system can be realized accurately and quickly, and adjusted through optimizing program, which is presented according to current values of front and rear stators.

Energy Control of Asynchronous Motor. A high-quality voltage controller is indispensable for the better energy-saving performance of the motor. After the output from the optimizing module, voltage value should be tracked through a controller. Under the voltage, stator current value should be stabilized as early as possible. Optimizing module is closely related to the accuracy of stator current. Therefore, a high-quality voltage controller is necessary for the energy-saving system of the motor.

Fig. 1 is the energy-saving control system of three-phase asynchronous motor. Within the optimizing interval $[\alpha, \beta]$, online optimizing module searches for the current point of the minimum stator, and exports the optimizing voltage value $\dot{U}$. The difference between $\dot{U}$ and $U_{a b}^{\prime}$ (voltage of low-pass filtering module) is indicated with $\Delta U$. After the input of $\Delta U$, voltage control module will regulate and control firing angle $\theta$ signal. After the input of $\theta$ signal, the voltage regulating module will call out $U_{\text {out }}$. Relative voltage signal should be supplied for the normal operation of voltage regulating module. Stator current $I_{a}$ of the motor can also be detected by relevant modules, and $I_{a}^{\prime}$ can be obtained accordingly. Digital average value sampling module should be employed to turn $I_{a}^{\prime}$ into signal. After the input of $I$ into the optimizing module, the new optimizing voltage $\dot{U}$

$\dot{U}$ can also be obtained through the optimizing program. After the process is repeated again and again, the voltage value $\dot{U}$ is approximate to the optimum energy-saving point. High-frequency signal is filtered through low-pass filtering module. The fluctuation of stator current signal is reduced to guarantee the correctness of input signal sampling of online optimizing module. 


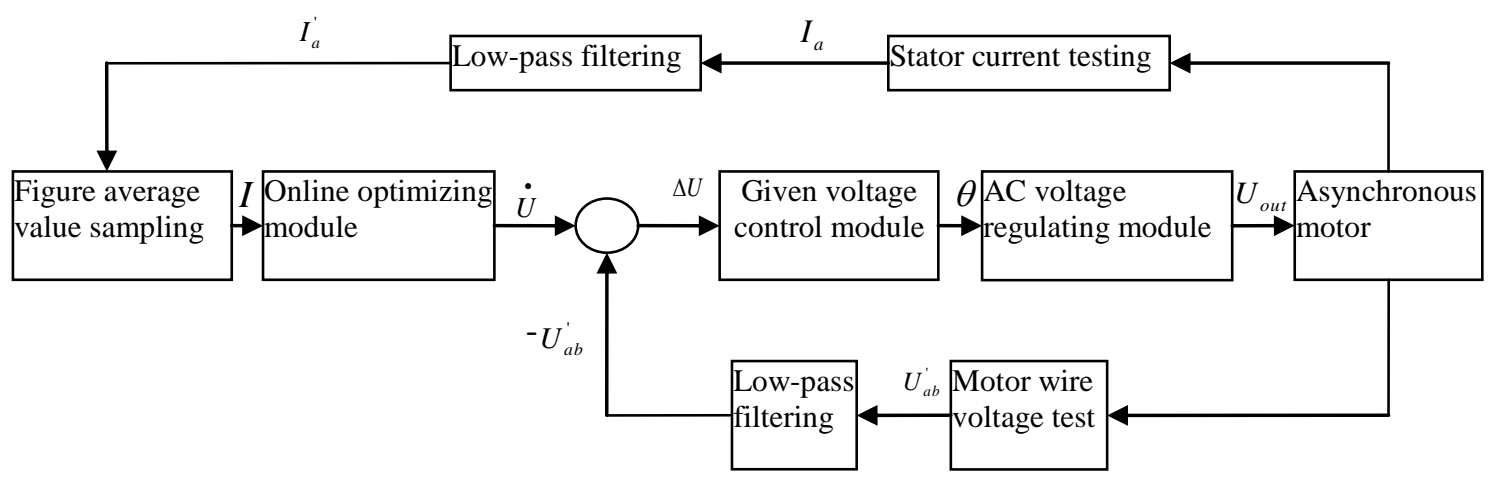

Figure 1. Energy-saving Control System of Three-phase Asynchronous Motor

\section{Automatic Minimum Energy-Saving Optimizing Control}

Three-phase AC Voltage Regulating Circuit. Phase-controlled three-phase AC voltage regulating circuit is adopted in the research. The symmetrical three-phase AC power is connected with three single-phase AC voltage regulating circuits, and phase difference among power supplies is $2 \pi / 3$. Three-phase AC voltage regulators are controlled in the neutral line-free star-like form, and their characteristics are distinct. The loads of all phases are mutually connected, thus forming loop current. Only thyristors of two different phases are connected, current can pass through the load. Therefore, such thyristors should have the trigger pulse of broad pulse or double-narrow pulse. The paper adopts the three-phase AC voltage regulator of neutral line-free star-like connection, and the voltage regulating model includes six thyristors. In voltage regulating circuit, VT1 and VT2, VT3 and VT4, and VT5 and VT6 are connected in pairs reversely. The trigger module produces trigger signal, and constant module presents firing angle. $F_{c n}$ module is added in the paper for the input of firing angle in the form of angle, indicated by $U_{\text {out }}=10 \times \alpha / 180$, where $\alpha$ refers to the firing control angle. Fig. 2 is a three-phase AC voltage regulator.

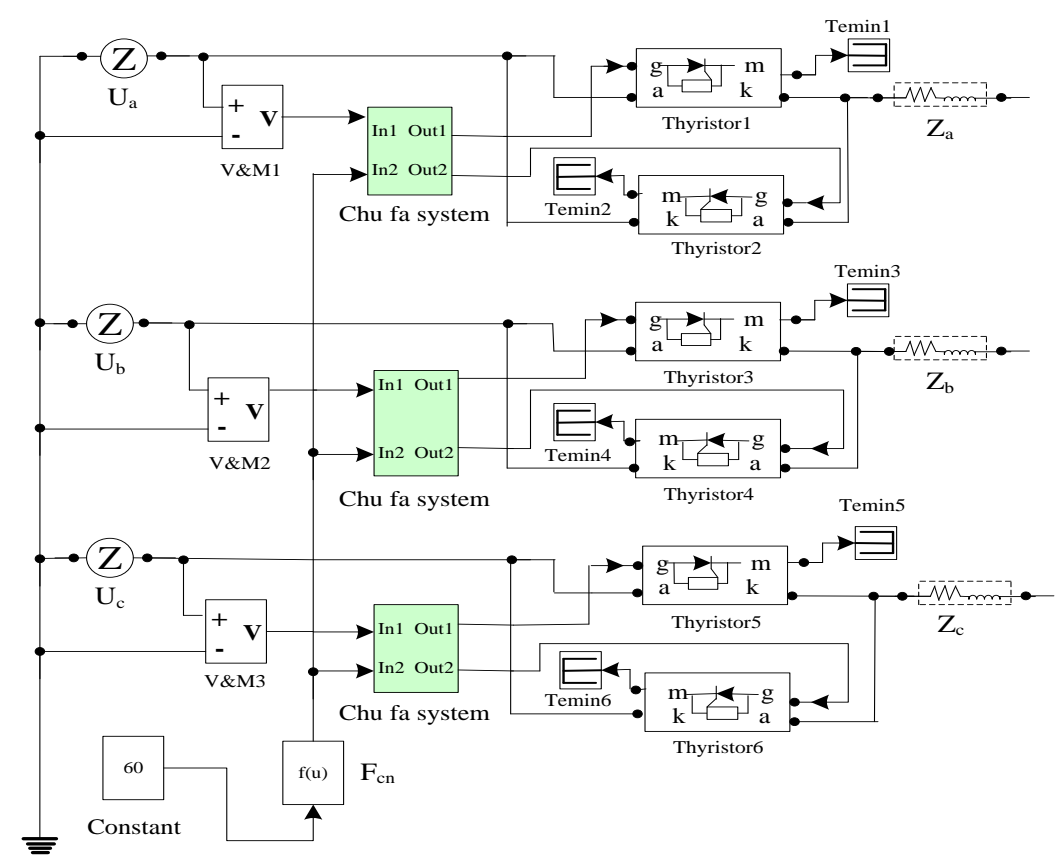

Figure 2 Three-phase AC Voltage Regulator

Determination of Optimum Efficiency $\eta_{j}$. In the full working scope, the optimum power factor of asynchronous motor changes in linear form. As for asynchronous motors of the same 
specification but supplied by different manufacturers, their power factors are discrete, and under the same working condition, their power factors are discrete as well. The expected optimum voltage regulating value is determined with $\eta_{j}=\eta_{\max }$ as the efficiency objective. Under different loads, there is an optimum value of supply voltage, which is jointly determined by the optimum efficiency $\eta_{j}$ and power factor $\cos \varphi_{j}$ of the system.

Under a load, $P_{2}=\eta_{j} P_{1}=m I_{1} U_{1 j} \cos \varphi_{j}$. If load rate $\beta=\frac{P_{2}}{P_{2 N}}, P_{2 N}=\eta_{N} P_{1 N}=m I_{1 N} U_{1 N} \eta_{N} \cos \varphi_{N}$ under the given load, so $P_{2}=\beta P_{2 N}=m I_{1 N} U_{1 N} \eta_{N} \beta \cos \varphi_{N}$.

Based on $m I_{1} U_{1 j} \eta_{j} \cos \varphi_{j}=m I_{1 N} U_{1 N} \eta_{N} \cos \varphi_{N}$, it can be derived that $U_{1 j}=\frac{I_{1 N} U_{1 N} \eta_{N} \cos \varphi_{N}}{I_{1} \eta_{j} \cos \varphi_{j}}$.

Set the efficiency objective $\eta_{j}=\eta_{\max }$, the optimum regulating voltage of a load rate is indicated by Eq.1:

$$
U_{1 j}=\frac{I_{1 N} U_{1 N} \beta \cos \varphi_{N}}{I_{1} \cos \varphi_{j}}=\frac{I_{1 N} U_{1 N} \cos \varphi_{N}}{\cos \varphi_{j}} \times \frac{\beta}{I_{1}}
$$

Automatic Minimum Energy Consumption Optimizing and Control System. Fig. 3 is the automatic minimum energy consumption optimizing and control system of three-phase asynchronous motor, which does three jobs: finishing the smooth startup of asynchronous motor in the mode of soft start after the system is electrified; offering the minimum energy consumption optimizing and control power factor according to the program controlled power factor; and carrying out closed-loop control of constant power factor after the determination of the optimum power factor. In reality, the system adopts LPC932 microcontroller, ATT7022A feedback channel, and LPC932singlechip interface. At last, the power supply volume of the motor is calculated and tested.

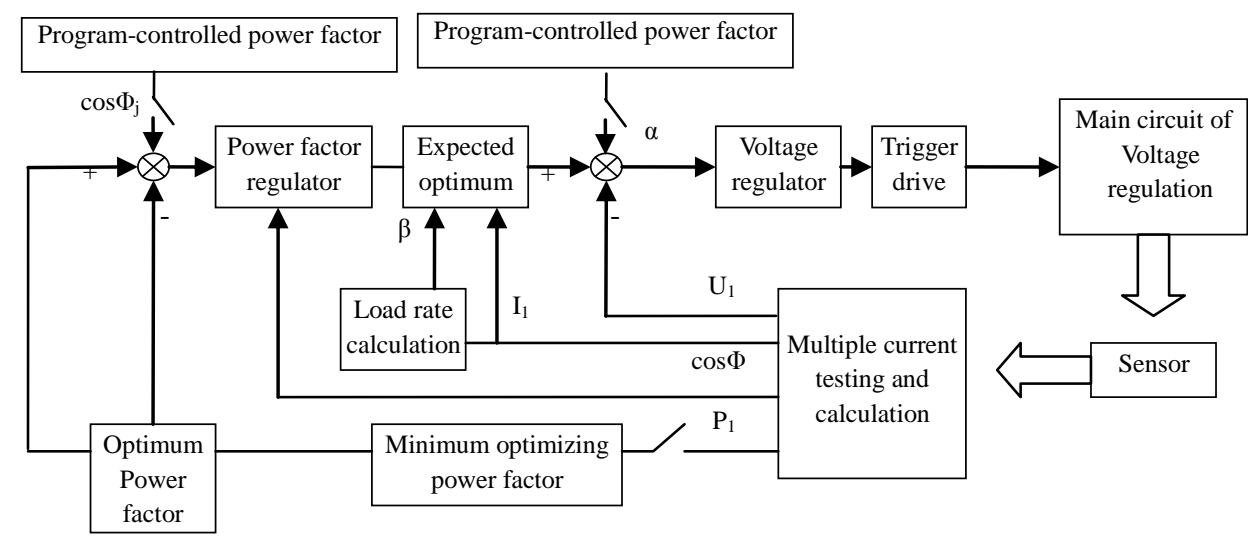

Figure 3. Minimum Energy-saving Optimizing and Control of Three-phase Asynchronous Motor

\section{Simulation Experiment}

LPC932 controller is adopted for the optimizing and simulation of automatic matching zoning of motor energy-saving control system. $5.5 \mathrm{KW}$ three-phase asynchronous motor is selected, with the rated voltage being $380 \mathrm{~V}$, rated current $11.8 \mathrm{~A}$, and rated revolution $1450 \mathrm{r} / \mathrm{min}$. The system carries out voltage regulating control within $0.5 \mathrm{~s}$ of simulation time after the start of motor, and stops voltage regulation after the stable operation of the motor. Parameter simulation results of the motor are shown as Fig. 4, Fig. 5, and Fig. 6: 


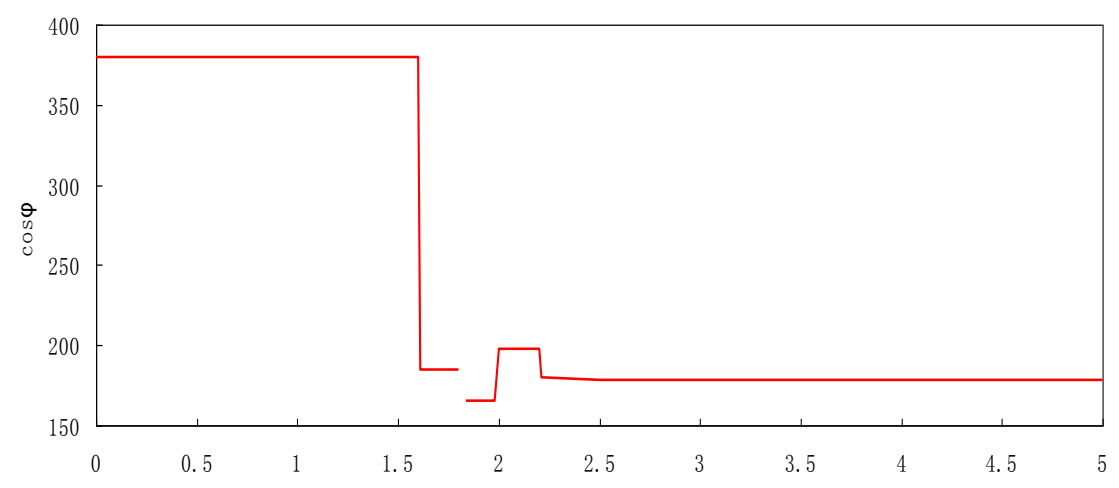

Figure 4. Output Voltage of Online Optimizing Model

Fig. 4 indicates the output voltage of online optimizing model of the motor. The online optimizing model makes the optimizing response after 1.61s. After 1.0s online optimizing, namely at about $2.61 \mathrm{~s}$, the output voltage of the module is $178 \mathrm{~V}$, being stable basically. Fig. 5 shows that the optimizing interval of the module is $[160 \mathrm{~V}, 220 \mathrm{~V}]$.

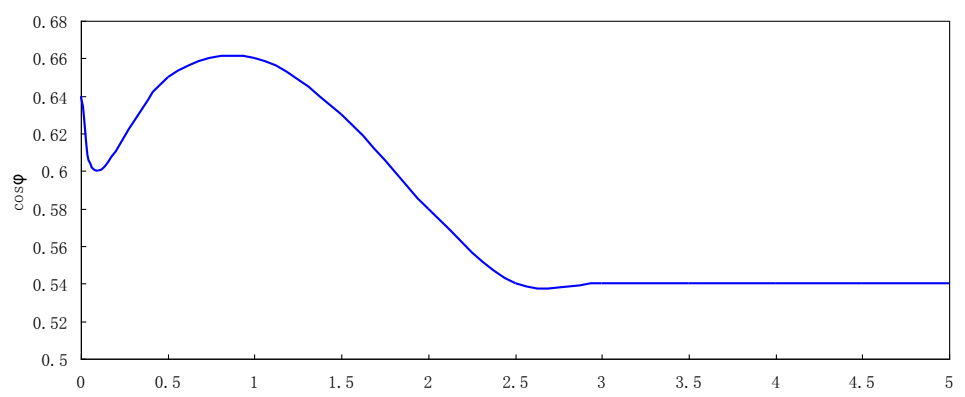

Figure 5. Power Factor of the Motor with Energy-saving Module

Fig. 5 is the power factor diagram of the motor with energy-saving model. Before $1.36 \mathrm{~s}$, the motor is close to the rated load, under which power factor is about 0.66 , rising slowly. After $1.36 \mathrm{~s}$, power factor does not decline sharply, but remains above 0.54 stably.

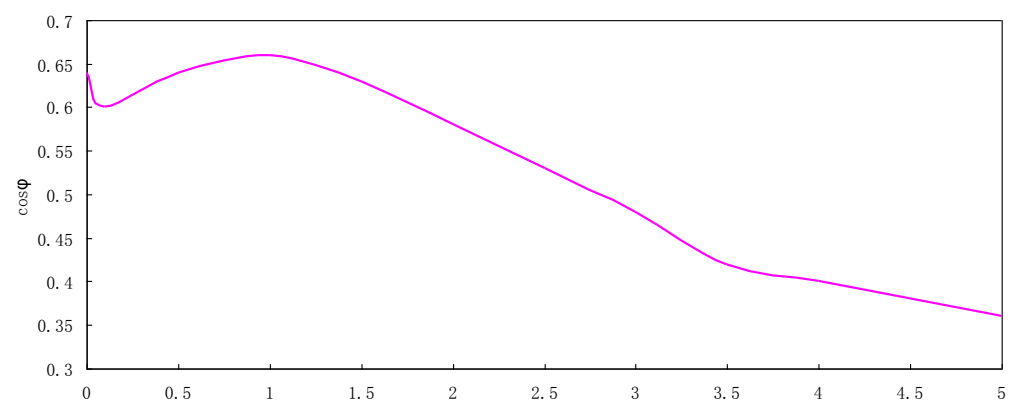

Figure 6. Power Factor of the Motor without Energy-saving Module

Fig. 6 is the power factor diagram of the motor without energy-saving module. Before 1.36s, Fig. 6 is roughly identical with Fig. 5 . After $1.36 \mathrm{~s}$, power factor starts to decline. At $5 \mathrm{~s}$, power factor drops to 0.36 .

Simulation results show that the active power consumed by the energy-saving system of the motor is calculated to be $842.2 \mathrm{~W}$, and declines to $264.3 \mathrm{~W}$ after the installation of the optimizing energy-saving module, so the energy-saving rate of the system is $68.5 \%$.

\section{Conclusions}

The energy-saving optimization of three-phase asynchronous motor is studied in this paper through the analysis of its working principle and structure. Via a three-phase AC regulator of neutral 
line-free star connection, six thyristors VT1 and VT2, VT3 and VT4, and VT5 and VT6 of the regulating model are connected reversely in pair so as to determine the optimum efficiency $\eta_{j}$ and power factor $\cos \varphi_{j}$, and further produce the technique of realizing the minimum energy consumption and the optimum voltage regulation and control through the automatic minimum energy consumption optimizing control system. The simulation results show that the optimization interval is $[160 \mathrm{~V}, 220 \mathrm{~V}]$, and the energy saving rate is lifted to $68.5 \%$ after the installation of the optimizing and energy-saving module.

\section{References}

[1] J.H.Zhang: Electric Manufacturing, (2013)No.6,p.47-49.

[2] W.J.Chu: Shanghai Medium and Large Electrical Machines, (2015)No.1,p.32-36.

[3] K.Chen, X.Y.Han and X.M.Li: Shanghai Medium and Large Electrical Machines, (2016)No.1,p.21-23.

[4] L.M.Guo: Journal of Henan Science \& Technology, (2014)No.12,p.85.

[5] T.Zhang, X.L.Zhang and S.T.Lu: Computer Simulation, Vol.32(2015)No.8,p.265-271.

[6] J.X.Ge: Technological Development of Enterprise, Vol.32 (2013), 32No.9,p. 102-104.

[7] X.Z.Gu, H.F.Xu and H.Q.Gu: Explosion-proof Electric Machine, Vol.50 ( 2015), 50 No.5,p.8-12.

[8] H.Xiao, H.M.He and S.K.Yin: Motor Technology, (2014) No.3,p. 97-99.

[9] K.J.Sun, Z.J.Wang and X.M.Shang: Motor Maintenance Technology Quick Check Permanent Manual(Chemical Industry Press, China 2016),p.31-34.

[10] Y.J.Zhang: Asynchronous Motor Frequency Control Technology(Mechanical Industry Press, China 2016),p.103-107. 